\title{
El Primer Coloquio de Directores de Centros de Restauración de América Latina, La Habana, Cuba, a 25 años
}

\author{
Salvador Díaz-Berrio Fernández
}

- n 1984, se inició y apoyó una serie de actividades para desarrollar las la-

E bores del Instituto Nacional de Antropología e Historia (INAH) tanto en el ámbito nacional como en el internacional. En el primero, lo más importante fue, por una parte, el impulso para establecer una nueva Ley Orgánica del instituto, a través de una comisión que formularía este documento; por la otra, en materia del patrimonio cultural inmueble, se llevaron a cabo en el Museo Nacional de Antropología la primera y la segunda reuniones para definir una Política de Conservación de Monumentos Históricos. Tuve la oportunidad de participar activamente en estas labores, como miembro de la comisión para formular la ley y como coordinador de la segunda reunión mencionada.

En el ámbito internacional, también tuve la ocasión de intervenir, a partir de ese año y con apoyo del INAH, como representante de México en el Consejo del Centro Internacional de Estudios para la Conservación y la Restauración de los Bienes Culturales (ICCROM-UNESCO); también como coordinador del equipo para elaborar la Lista Indicativa de sitios de México para su inscripción en la Lista del Patrimonio Mundial de la UNESCO; asimismo, designado por el director general de este organismo, como experto en la Campaña Internacional para La Habana Vieja y, por último, en calidad de representante de México y del ICCROM, en el Primer Coloquio de Directores de Centros de Restauración de América Latina, motivo de este comentario.

A la reunión de La Habana asistieron delegados de nueve países latinoamericanos: Argentina, Bolivia, Cuba, Ecuador, Perú, Venezuela, Brasil, Panamá y México. También se contó con la participación de seis organismos internacionales: el Consejo Internacional de Museos (ICOM), el Consejo Internacional de Monumentos y Sitios (ICOMOS), el Instituto Iberoamericano de Cooperación Cultural, la representación latinoamericana del Programa de las Naciones Unidas para el Desarrollo (PNUD-UNESCO), la UNESCO a través de su Oficina Regional de Cultura para América Latina y el Caribe con sede en Cuba -en su carácter de organismo huésped-, y el ICCROM, cuyo director estuvo como invitado especial.

Tomó parte un solo especialista de las primeras seis naciones, incluyendo a Cuba, así como dos de los sendos países de Brasil y Panamá. México fue representado por cuatro especialistas del INAH: el director general, doctor Enrique Florescano (también como invitado especial); el director de la Escuela Nacional de Conservación, Restauración y Museografía (ENCRyM), profesor Jaime Cama; el director de Restauración del Patrimonio Cultural (DRPC), licenciado Agustín Espinosa, y el jefe de Proyectos Técnicos de la Secretaría Técnica, doctor Salvador Díaz-Berrio. Como sucede en este tipo de reuniones, participaron como 
observadores nueve especialistas del país huésped, cinco funcionarios de la oficina de la UNESCO en La Habana y cinco más de la Comisión Nacional Cubana para la UNESCO.

Independientemente de la importancia de diversas aportaciones individuales, la de mayor interés fue el documento colectivo de conclusiones y recomendaciones, del que cabe destacar cinco grandes temas:

1) Lograr mayor intercambio de experiencias entre centros e instituciones nacionales de restauración, mediante la coordinación de la Oficina Regional de la UNESCO para América Latina y el Caribe.

2) Incrementar programas de formación de personal técnico en las instituciones especializadas de la región, con la colaboración de organismos internacionales, asimismo especializados.

3) Buscar mecanismos de financiamiento, tanto regionales como internacionales, para reforzar con personal, equipos y materiales las instituciones nacionales y regionales de formación especializada.

4) Elaborar un inventario de recursos de la región -humanos, técnicos y científicos- para lograr su óptima utilización y mantener un flujo de información y publicaciones en español entre instituciones establecidas en ella.

5) Estimular investigaciones sobre materiales y procesos técnicos de conservación y restauración entre los centros de la región, utilizando y ampliando los programas de becas nacionales e internacionales disponibles.

Además, se expresó la necesidad de dar continuidad a los proyectos regionales del PNUD-UNESCO y se insistió especialmente en la conveniencia de "restablecer el carácter regional que tuvo el Centro de Churubusco en México, por el papel pionero que desarrolló y por la infraestructura con que cuenta".

En su ponencia, el entonces director de la ENCRyM afirmó: "Hay pocos

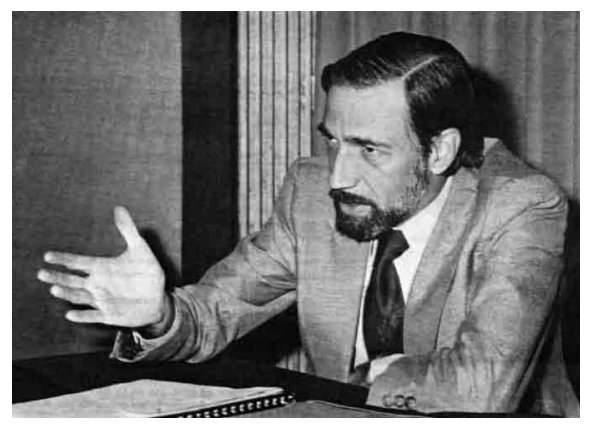

Retrato del doctor Salvador Díaz-Berrio Fernández en 1980. (Cortesía del autor).

países de América Latina y el Caribe que no hayan enviado personal a capacitarse en restauración a la Escuela de México; por ello, esta reunión nos pareció particularmente significativa para establecer el punto de partida de una política". Justamente, este objetivo prioritario, detallado en los cinco temas mencionados más arriba, es lo que todavía no se ha logrado concretar.

A pesar de los propósitos expresados en esta reunión -la primera y la última de este tipo-, los deseos y declaraciones en torno de este punto de partida de una política regional en materia de formación y trabajo en el campo de la conservación y la restauración del patrimonio cultural no se han cumplido. Tampoco se ha restablecido el carácter regional del Centro de Churubusco, a pesar de que ha mantenido su condición de pionero en diversos temas y que la ENCRyM cuenta ahora con una infraestructura mucho más amplia.

Por este motivo, 25 años después, la reunión realizada en La Habana y el texto de sus conclusiones y recomendaciones representan gran importancia por su contenido y propósito, así como por su carácter esencial, en esta materia. Adicionalmente tienen gran interés por su valor histórico: no sólo muestran el avance alcanzado en este tema en aquel tiempo -que será útil comparar con nuestra situación actual-, sino también llevan a preguntarse por qué no se ha proseguido en la dirección entonces señalada.

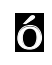

Nota del editor

El "Informe del primer coloquio de directores de centros de restauración de América Latina", publicado originalmente en 1984 en Restauración -boletín de la entonces Dirección de Restauración del Patrimonio Cultural del Instituto Nacional de Antropología e Historia (INAH)-, merece su republicación en esta sección dedicada al rescate y la reflexión de nuestra memoria disciplinar, por cuando menos, tres motivos:

- El primero de ellos, para ampliar la difusión de un documento resultado del debate, el intercambio y el consenso profesionales; sobresaliente por haberse gestado en una porción del mundo caracterizada por peculiaridades, contextos y desarrollos profesionales en el ámbito patrimonial que son únicos en el globo.

- El segundo, porque responde a la necesidad de explorar nuestro devenir histórico -cuyos detalles a veces sólo conocen sus protagonistas- desde una mirada fresca; un ejercicio reflexivo que en la actualidad para nada es inútil, ya que hace ver lo logrado, más allá de la miopía propia de los inmediatismos y valorando los posibles efectos.

- El tercero, por supuesto, se debe a la vigencia de las reflexiones producidas hace 25 años: un ejercicio de análisis que no obedece a la nostalgia, sino que plantea la responsabilidad de tomar acciones, a veces remontando caminos, quizá no siempre sostenidos con persistencia.

Esta trilogía de argumentos es ampliada y profundizada por el doctor Salvador Díaz-Berrio Fernández, participante del coloquio, quien cuenta con una amplia y reconocida trayectoria en el campo de la conservación patrimonial tanto en México como en el extranjero. Así, desde una perspectiva informada, aguda y, sobre todo, profunda, el doctor Díaz-Berrio plantea disyuntivas sobre la disciplina de la conservación-restauración latinoamericana y el papel que México ha ocupado en su acaecer, lo que indiscutiblemente conlleva reflexionar sobre la función que deberá asumir como parte de su responsabilidad histórica. 
Informe del Primer Coloquio de Directores de Centros de Restauración de América Latina

\section{INTRODUCCIÓN}

El Primer Coloquio de Directores de Centros de Restauración de América Latina, celebrado en La Habana, Cuba, del 23 al 26 de noviembre de 1984, organizado por la Oficina Regional de la UNESCO para América Latina y el Caribe, ha tenido como propósito hacer un análisis de la situación en la región y del papel de los centros de restauración existentes, en relación con las necesidades y condiciones de cada país, teniendo los siguientes objetivos: proporcionar un primer diálogo entre las instituciones y centros de restauración y organismos internacionales; recomendar el establecimiento de una red regional de coordinación y cooperación e intercambio de experiencias; recomendar lineamientos tendentes a estructurar un programa cultural coherente a nivel nacional y regional en materia de conservación.

Participaron representantes de Argentina, Bolivia, Brasil, Cuba, Ecuador, México, Panamá, Perú y Venezuela, así como de organizaciones internacionales especializadas y un ponente de Polonia.

\section{CONCLUSIONES Y RECOMENDACIONES}

Hubo consenso entre los participantes al Primer Coloquio de Directores de Centros de Restauración de América Latina en lo relativo a las siguientes necesidades expresas:

1. Establecer mecanismos que permitan una mayor comunicación, coordinación e intercambio de experiencias entre los centros de restauración e instituciones nacionales de la región.

2. Con la colaboración de instituciones internacionales especializadas y dentro de estrategias previamente estudiadas para la región, incrementar los programas de formación de personal técnico y administrativo en las instituciones especializadas de la región.

3. Buscar mecanismos de financiamiento regionales, internacionales y de fuentes alternas, que permitan reforzar con personal capacitado, equipos y materiales, las instituciones nacionales y/o instituciones con vocación regional especializadas en el campo de la conservación y restauración.

4. Elaborar un inventario de los recursos humanos, técnicos y científicos de la región para permitir su óptima utilización.
5. Estimular investigaciones entre los centros sobre materiales y procesos técnicos de conservación y restauración, inherentes a cada región.

6. Que los responsables de los centros de restauración y conservación realicen esfuerzos para que sus gobiernos incrementen las acciones de conservación y restauración y de capacitación de personal especializado.

7. Que los organismos internacionales e instituciones internacionales especializadas incrementen sus programas en América Latina y el Caribe, relativos a la salvaguardia del patrimonio cultural.

8. Que los gobiernos de la región absorban en sus programas de restauración y conservación a personal especializado y lo destinen a cargos de responsabilidad en su respectiva competencia.

En la sesión plenaria se presentaron cinco resoluciones por las cuales se crea un grupo de trabajo asesor de la Oficina Regional de Cultura para América Latina y el Caribe, se recomienda a las instituciones internacionales y a las nacionales, adopten medidas que aseguren un óptimo aprovechamiento de las becas que ofrecen para formación de personal especializado; se recomienda poner en marcha programas de publicación y difusión de material técnico y especializado tanto de la UNESCO como de los países, en los campos de la conservación y la restauración; se recomienda a las autoridades mexicanas concertar acuerdos con organismos e instituciones internacionales para restablecer el carácter regional que tuvo Churubusco, y finalmente, se apoya la continuidad de los proyectos PNUD/UNESCO.

\section{RESOLUCIÓN NÚM. 1}

I. Considerando que la cooperación entre los países de América Latina y el Caribe y sus relaciones con organismos internacionales especializados son elementos fundamentales para el desarrollo de programas de conservación y restauración del patrimonio cultural.

II. Considerando que pese a los esfuerzos realizados existe poca coordinación entre los programas que desarrollan las distintas organizaciones regionales e internacionales e instituciones especializadas en la región y que se hace necesario una mejor utilización de los recursos disponibles para programas de cultura y de obtención de fuentes alternativas de financiación.

III. Considerando que para obtener una óptima cooperación regional e internacional es necesario establecer los mecanismos adecuados. 
1. Constituir un Grupo de Trabajo Técnico encargado de asesorar a la Oficina Regional de Cultura de la UNESCO para América Latina y el Caribe en el estudio de la situación existente en los distintos países en el campo de la conservación y restauración del patrimonio cultural con el fin de identificar, evaluar y planificar programas anuales de acción para atender las necesidades prioritarias de la región.

2. Solicitar a la Oficina Regional de Cultura de la UNESCO para América Latina y el Caribe, que convoque anualmente al Grupo de Trabajo Técnico Asesor, bien sea en su sede o fuera de ella, e indique a las instituciones internacionales especializadas, tales como el ICCROM, ICOMOS, ICOM y demás que considere conveniente, incluyendo las instituciones de financiamiento (PNUD, BID, Banco Mundial, OEA, etc.) e instituciones regionales (OEA, Andrés Bello, etc.) a fin de desarrollar conjuntamente el plan de acción propuesto.

3. Solicitar a la Oficina Regional de la UNESCO para América Latina y el Caribe establecer la coordinación necesaria entre los centros nacionales de restauración y los organismos regionales e internacionales e instituciones especializadas que desarrollen programas culturales en la región, con miras a la óptima utilización de los recursos humanos y financieros disponibles.

4. Solicitar a las instituciones nacionales y regionales especializadas cooperar con la Oficina Regional de Cultura de la UNESCO para América Latina y el Caribe, en los planes de coordinación regional e intercambio de experiencia e información.

5. Solicitar a la Oficina Regional de la UNESCO para América Latina y el Caribe transmitir la presente resolución a las distintas organizaciones internacionales y regionales e instituciones especializadas con miras a establecer dicha coordinación.

\section{RESOLUCIÓN NÚM. 2}

I. Considerando que uno de los factores que dificulta la adecuada protección del patrimonio cultural es la escasez de recursos humanos especializados y que una de las necesidades apremiantes de la región es precisamente la capacitación de dicho personal.

II. Considerando que los recursos disponibles para programas de capacitación deben ser utilizados en forma que aseguren su mayor rendimiento.
1. Recomendar a las instituciones internacionales especializadas, mantener informada a la Oficina Regional de Cultura para América Latina y el Caribe, de sus programas de capacitación y de tomar en cuenta en el otorgamiento de becas los planes regionales existentes y las necesidades prioritarias de la región y solicitar que en la medida de lo posible desarrollen sus programas en instituciones especializadas de América Latina y el Caribe.

2. Recomendar a las instituciones internacionales especializadas considerar las solicitudes de becas que hayan sido avaladas por los respectivos centros de restauración.

3. Solicitar a la Oficina Regional de Cultura de la UNESCO para América Latina y el Caribe transmitir la información pertinente, a través de su Centro de Documentación, a las instituciones especializadas de la región.

4. Recomendar a instituciones nacionales especializadas que ofrezcan programas de capacitación a los demás países de la región, estableciendo mecanismos apropiados para la selección de candidatos.

5. Solicitar a la Oficina Regional de Cultura para América Latina y el Caribe que comunique la presente resolución a las instituciones especializadas regionales e internacionales que ofrezcan programas de capacitación en los campos de restauración y conservación.

\section{RESOLUCIÓN NÚM. 3}

I. Considerando que una de las necesidades manifestadas por los participantes en el presente Coloquio es la de mantener un flujo de información entre sus instituciones especializadas y las instituciones internacionales competentes en el campo de la restauración, conservación y salvaguardia del patrimonio cultural.

II. Considerando que existe una necesidad en la región de material especializado en técnicas de conservación, restauración y salvaguardia del patrimonio cultural.

\section{Resuelve}

1. Recomendar a la Oficina Regional de Cultura para América Latina y el Caribe que con asistencia y colaboración de instituciones nacionales e internacionales especializadas, publicar y difundir en idioma español, a través de su Centro de Documentación, 
el material técnico y especializado publicado por la UNESCO en otras lenguas, sobre conservación, restauración y salvaguardia de bienes culturales y otros que considere relevantes, e igualmente publicar y divulgar el informe con las conclusiones, recomendaciones, resoluciones y ponencias presentadas por los participantes en el Primer Coloquio de Directores de Centros de Restauración de América Latina.

2. Solicitar al Comité del Patrimonio Mundial (Convención concerniente a la Protección del Patrimonio Mundial Cultural y Natural, 1972, Art. 22) atender las peticiones que presenten los países de la región, signatarios de la convención para dar cumplimiento a las acciones contenidas en la presente resolución.

3. Solicitar a la Oficina Regional de Cultura para América Latina y el Caribe transmitir esta resolución a las instituciones nacionales e internacionales especializadas y al Fondo de Patrimonio Cultural de la UNESCO.

\section{RESOLUCIÓN NÚM. 4}

I. Considerando que es una necesidad prioritaria de la región la capacitación de personal especializado en la conservación y restauración de los bienes muebles e inmuebles del área.

II. Considerando el papel pionero desarrollado por el Centro de Restauración de Churubusco (México) en la capacitación de personal especializado en la región y que la infraestructura con que cuenta permite incrementar esta formación.

\section{Resuelve}

1. Solicitar a las autoridades mexicanas hacer lo procedente con el fin de concertar acuerdos con instituciones internacionales y regionales tales como la UNESCO, PNUD, OEA, ICCROM, ICOMOS, ICOM y demás que considere pertinentes, para restablecer el carácter regional que tuvo Churubusco (México) hace algunos años.

2. Recomendar a los diferentes centros de restauración e instituciones especializadas nacionales, o de vocación regional, solicitar a sus autoridades respectivas, realizar acciones similares.

3. Recomendar a la Oficina Regional de Cultura de la UNESCO para América Latina y el Caribe transmitir la presente resolución a las instituciones internacionales y regionales mencionadas en el párrafo anterior.

\section{RESOLUCIÓN NÚM. 5}

Se recomienda la continuidad de los proyectos PNUD/ UNESCO en el ciclo de programación 1982-1986 y su continuidad en el programa quinquenal 1987-1991, a nivel nacional y regional, en apoyo a las actividades que realizan los países en el rescate y preservación de su patrimonio cultural y en la creación de centros de restauración.

El grupo técnico asesor de la Oficina Regional de Cultura de la UNESCO, quedó integrado por el Lic. Agustín Espinosa Chávez, Director de Restauración del Patrimonio Cultural del INAH (México), por el Lic. Froilán Argandoña, Director del Instituto $\mathrm{Na}$ cional del Patrimonio Artístico (Bolivia), por el Prof. Reginaldo di Piero, Director de Proyectos e Investigaciones del Centro de Sociología de Cultura, de la Universidad Cándido Méndez (Brasil) y por el Arq. Demetrio Toral de León, Director del Patrimonio Histórico y Director del Centro OEA/INAH (Panamá).

Finalmente, los participantes al Coloquio presentaron una moción de agradecimiento a la Oficina Regional de Cultura de la UNESCO para América Latina y el Caribe con sede en La Habana, Cuba, por los esfuerzos realizados para llevar a cabo el Primer Coloquio de Directores de Centros de Restauración de América Latina, que dio oportunidad a los participantes de establecer un diálogo regional entre los responsables de dichos centros e instituciones especializadas internacionales y nacionales, del cual se derivaron importantes conclusiones y recomendaciones que reflejan las necesidades y anhelos de la región. Asimismo, los asistentes expresaron su agradecimiento a las autoridades del gobierno de Cuba por la cordial acogida y la atención brindada.

A este Coloquio asistieron como representantes de México, el Director General del INAH, Dr. Enrique Florescano, el Director de Restauración del Patrimonio Cultural, Lic. Agustín Espinosa Chávez, el Director de la Escuela Nacional de Conservación, Restauración y Museografía, Prof. Jaime Cama Villafranca y el asesor de la Secretaría Técnica del INAH, Arq. Salvador Díaz-Berrio. Asimismo el Dr. Enrique Florescano ofreció el apoyo para realizar el Segundo Encuentro en México para finales de este año. 


\section{Resumen}

El presente artículo examina los resolutivos del Primer Coloquio de Directores de Centros de Restauración de América Latina, evento que tuvo lugar en La Habana, Cuba, en 1984. Además de contextualizar las circunstancias históricas que los vieron nacer, particularmente en relación con las labores realizadas por el Instituto Nacional de Antropología e Historia (INAH) a escala nacional e internacional, se reseñan las particularidades propias del coloquio, subrayando sus principales contribuciones. Estas aportaciones son analizadas en una visión retrospectiva sobre el papel que ha ocupado México en la formación de especialistas del campo de la conservación y restauración de la región latinoamericana, con vistas a una reflexión actualizada que exige responsabilidades por cumplir en el futuro.

\section{Palabras clave}

Conservación, América Latina, Coloquio, historia, análisis.

\section{Abstract}

This article examines the conclusions of the First Colloquium of Latin America Restauration Centers, which took place in 1984 at La Habana, Cuba. After contextualising the historical circumstances of their origins, particularly in relation to the activities carried out by the Instituto $\mathrm{Na}$ cional de Antropología e Historia (INAH) at a national and international scale, the present text studies the colloquium particularities, with an emphasis on its main contributions. These are retrospectively examined on regards of the role that Mexico has occupied for the training of specialists in conservation and restoration at Latin America, in order to bring up a fresh consideration to the current demands for fulfilling future responsibilities.

\section{Keywords}

Conservation, Latin America, Colloquium, History, Analysis. 